\title{
Beneficence in general practice: an empirical investigation
}

\author{
Wendy A Rogers The Flinders University of South Australia
}

\begin{abstract}
Objectives-To study and report the attitudes of patients and general practitioners (GPS) concerning the obligation of doctors to act for the good of their patients, and to provide a practical account of beneficence in general practice.

Design-Semi-structured interviews administered to GPs and patients.
\end{abstract}

Setting and sample-Participants randomly recruited from an age and gender stratified list of GPS in a geographically defined region of South Australia. The sample comprised twenty-one general practitioners and seventeen patients recruited by participating GPs.

Results-In practice, acting for the good of the patient not only accommodates the views of patients and GPs on expertise and knowing best, but also responds to the particular details of the clinical situation. Patients had a complex understanding of the expertise necessary for medical practice, describing a contextual domain in which they were expert, and which complemented the scientific expertise of their GPs. General practitioners identified multiple sources for their expertise, of which experience was the most significant. The role of the GP included responding to individual patients and particular clinical problems and ranged from the assumption of responsibility through to the proffering of medical advice. Conclusion-This study found that GPs acting for the good of their patients covered a variety of GP actions and patient preferences. Beneficence was not justified by presumed patient vulnerability or the inability of patients to understand medical problems, but furthered through a recognition of the different areas of expertise contributed by both parties to the consultation.

(Fournal of Medical Ethics 1999;25:388-393)

Keywords: Beneficence; general practice; empirical study

\section{Introduction}

The moral principle of beneficence is one of the time-honoured ethical foundations of medical practice. ${ }^{12}$ Acting beneficently towards another person means acting for the good of that person. ${ }^{3}$ In medical practice, this involves using specialised medical knowledge to advise patients with regard to their health; that is, doctors are morally obligeg to act for the good of their patients. At time 쟁 medical beneficence encompasses taking mucio responsibility for patients, for example in the treatment of acutely and severely ill people. A other times, medical beneficence has been the screen for acts of paternalism in which doctors make decisions for patients, justified on the grounds that, in this situation, the doctor knows the patient's good better than the patient does. $\frac{}{6}$ Neither total assumption of responsibility not paternalism adequately answers the question $\Phi$ how beneficence should be displayed in gener practice. Rather, the role of medical expertise is central to this question, because it is through a understanding of expertise that we may appreciate the ways in which the knowledge and experience of both patients and GPs contribute to acting fof the good of patients. This paper presents datit from a study which explored the ways in which patients and general practitioners understood the idea of medical expertise and acting for the patients' good in general practice, in order reach a more practical understanding of benefip cence.

\section{Methods}

The study consisted of two focus groups an thirty-eight individual in-depth interviews with GPs and patients. The GPs were selected by strat fied, purposive sampling from a master list of all GPs working in a geographically defined region if South Australia. The sampling strata, designed to maximise the range of GPs interviewed, included gender, age, years since registration, practice location (urban or rural), type of practice and number of sessions worked per week. Randomly selected GPs within each strata were contacted t] participate in the study, and requested to recruit $\frac{1}{8}$ patient for interview. Ten GPs recruited one patient each, three GPs recruited two patients each, angु eight did not recruit a patient. Low back pain was selected as the index condition as it is a commog problem, raising a number of interesting manage ment issues. 
The final sample comprised twenty-one GPs; ten female and eleven male, ranging in age from twenty-eight to seventy years. These GPs had between two and forty-six years experience in general practice and were located in a variety of geographical settings and practice arrangements. The patients in the study comprised twelve female and five male patients with a similar age range to that of the GPs. All of the patients but one were recruited by participating GPs, making it possible to match their data with that of their GP. During the audio-taped semi-structured interviews, participants were asked to describe the reason for seeing the GP and the events in the recent consultation, to indicate the important issues arising in this encounter and to discuss sources of knowledge, expertise and aspects of decision making in the consultation. The interviewer encouraged participants to explore each question in depth and participants were free to raise any further relevant issues during the interview.

The transcribed interviews were analysed using codes derived both theoretically and from close textual analysis. ${ }^{6-8}$ This resulted in four major categories. These were: beneficence-in-practice; choice and control; GP-patient relations, and trust. This paper reports on the category, beneficence-in-practice, which organises and describes material relating to the role of medical expertise in decision making between GPs and patients.

\section{Results}

PATIENTS' VIEWS ON BENEFICENCE-IN-PRACTICE The category of beneficence-in-practice encompasses the data from both patients and GPs. Within the patient data, the two dominant themes concerned the sources and the nature of medical expertise, and ideas about who knows best in the consultation.

Patients understood medical expertise to derive from several sources, including medical knowledge and experience:

"They know - that's their job and they know what to do." (Patient K) and "I suppose because he would think ... I mean, he's a doctor, he should, should know, shouldn't he?" (Patient M)

However, this medical knowledge and experience was augmented by information provided at the discretion of the patient. Over time (and most of these patients were in long term relationships of medical care with their doctors) GPs built up a stock of information about that patient such that some patients considered their GP more expert than a specialist, on the basis of this personal knowledge:
"I would probably trust her judgment quite a lot because I think that she would know me more than a specialist would and would know my situation better than he would or she would." (Patient G)

The experience of low back pain was seen to be an important source of expertise for both GPs and patients. For patients, previous experience of the same condition was a very powerful source of practical expertise in terms of recognising the problem and knowing what to do about it. Bodily knowledge was not limited to states of pain; patients were confident of their own physical experiences and were able to use these to augment or to counter the medical knowledge of the GP. However, this personal knowledge often required formal validation from a GP for the purposes of social recognition, such as sick certification.

A further source of expertise described by patients was that of their own self-knowledge with regard to their values and aims. This was very clearly articulated by one patient:

"I went trekking last year to Tibet and I know that it hurt my knees a lot ... . And it's damned annoying, but I chose to go to Tibet and I chose to do that trekking, and what it did for me in the whole sphere of my life has been worth the problem that I'm having with my knee." (Patient I)

Patients identified third party sources of information, such as the media, which could question or support the medical expertise of their GP. Three participants discussed the role of television documentaries in providing information which stimulated further discussions between patient and GP.

Building upon this understanding of medical expertise, it was then possible to explore the relationship between expertise and patients' attitudes towards knowing best in the consultation. Patient attitudes varied across a spectrum, from those who said that the doctor knew what was best for them through to those who felt that despite the doctor's medical expertise, patients knew what was best for themselves. This initially simple divide into those who believed doctors knew best and those who did not became progressively more complex; as with expertise, there were multiple facets to such an attitude.

All of the patients recognised the medical expertise of GPs, and for many patients this translated into the attitude that GPs knew what was best for them. For several patients, this was reinforced by successful treatment of their problem:

"Well my doctor just said that he, you know, thought that physio would be the way to go and I just went along with him. As far as I'm concerned 
he knows what he's talking about, which is proving right." (Patient B)

Several of the patients held an attitude of joint expertise, in which they recognised the importance and value of GPs' medical knowledge, but also discussed the significance of their own expertise in assessing their problem:

"I suppose really it's up to you to get your point across, about how you are feeling." (Patient F)

Sometimes this joint expertise required the patient's input into practical matters, such as management decisions contingent upon the availability of money or time; matters within the contextual domain. Joint expertise was built up by a process of dialogue, with both GP and patient contributing information and expertise to arrive at a position of knowing best in this case.

A smaller group of patients described themselves as knowing best. Again, the underlying reasons were not uniform. One patient, whilst recognising the expertise of doctors, did not translate this into an overall view that doctors know best:

"I don't really like to be told that I have to do this because this person's a doctor and they know more than me. I accept a doctor's expertise but I still think it's my body and I need to have an informed - make informed choices about what I'm going to do and I'm the only one that can really weigh up what my life is about and how important it is for me to make those kind of decisions." (Patient I)

Other patients saw themselves as knowing best by default; over lengthy periods of time doctors had been unsuccessful with either diagnosis or treatment, leaving the patient as the one who knew best in terms of managing his or her pain.

This data from the patients provides an insight into the kinds of expertise relevant in the consultation, and the ways that patients think about GPs' roles, in terms of knowing best.

\section{GPS' VIEWS ON BENEFICENCE-IN-PRACTICE}

With regard to medical expertise, GPs identified several sources contributing to their management of low back pain. These were, in order of frequency: professional experience; reading journals or books; specific educational material from the state work insurance organisation; personal experience; continuing postgraduate medical education, and discussions with colleagues. Experience was cited most frequently as an important source of expertise in the approach of these GPs to managing low back pain. Professional experi- ence was a combination of familiarity throug exposure to many cases coupled with pragmatic approaches to treatment. Theoretical knowledge about specific treatments such as anti inflammatory drugs or analgesics was tempere by the GPs' experiences of what had worked similar cases in the past.

\section{Personal experience}

As well as experience in the broad sense of seein many different patients, GPs also cited experiences with particular patients as contributing to thein expertise in specific cases. Reaching a diagnosid was felt to be easier in patients who were known the GP. In patients with a past history of the samg condition, GPs could draw upon both their own and the patients' knowledge of successfull strategies, as well as tailoring information towards the known preferences of the patients.

Personal experience of low back pain was cit by five GPs as playing an important role in their management of patients. This experience workeg in a number of ways, increasing the GPs' compassion and insight as well as altering specifa management strategies, such as length of time resting in bed, use of analgesics and phys otherapy, and expectations about long term bas care:

"Ah, well I think you tend to appreciate - I thin diagnostically it's helped me a bit and I think als़ in sympathy I guess. You know having bee⿳⺈ through it ... I was off work for a couple of week and had visions of never working again and so you know I really ... I was in a pretty bad way aboutyear ago." (GP H)

The next source of expertise identified by GPs w education, which took various forms, includir formal events such as seminars and workshops reading books and journals or the use of protocols. The various contributions of education versus experience were difficult to determine:

"You know, you read journals and stuff and the idea of looking at low back pain I guess has changed a bit over the years, but how much of that is education that I've absorbed from things I' been to and journals and how much of it is just thfe passage of time and having seen enough people. guess, I suspect it's more the passage of time $\stackrel{\circ}{\circ}$ rather than actual educational things that have been aimed at me." (GP I)

Some GPs recognised the practical expertise of patients in various areas. When dealing widi patients who had previous experiences of low bae pain, the opinion of the patient was usuals 
accepted, particularly in the context of a longstanding relationship:

"I'm quite often swayed by the patient when determining when they are ready to go back to work because ... people often do have a fairly good idea of what they can do with their current state of pain." (GP U)

The issue of medical expertise overlapped with observations about the role of GPs. First, GPs identified their role in supporting patients and in helping them to further their own agendas. In this role, medical expertise was used to facilitate the aims of the patient; this expertise was significant but not called upon as a reason to justify decisions. A second role described by GPs was that of educator:

"It's more of an education attitude ... I talk about the tissue elements that comprise the back, the bones, the ligaments, the muscles. I talk about the importance of the soft tissues in supporting the bones rather than vice versa which is usually interesting to the patient ... and then talk about the importance of strength, fitness and flexibility in those soft tissues in maintaining the health and function and comfort of the overall back unit." (GP G)

A third role described by GPs was that of taking charge, because of their expertise. In this role, medical expertise justified the GP's command of the situation:

"I do what I think is right, and I explain to them why what they think isn't right ... . What the patient wants isn't necessarily what ... is going to be good for the patient is it? I mean, patients are not skilled in medical diagnosis." (GP K)

Building upon ideas of expertise and GP roles, it was possible to identify a range of GP attitudes towards the idea of knowing best in the consultation. Again, it is important to emphasise that these attitudes are not rigid in that there were many factors which influenced GPs' approach to their own knowledge and the place of this knowledge in the context of treating a specific patient. Unsurprisingly, few of the GPs held the attitude that patients knew better than they with regard to diagnosis as well as management, however a minority of GPs expressed the following sentiments:

"We're not experts. We're definitely not experts on the human body I don't feel." (GP T)

The majority of GPs described knowing best as a joint enterprise in which they contributed their medical knowledge and this was complemented by the personal knowledge of the patient. These
GPs used their knowledge to inform and guide the patients in the areas in which they felt expert, usually diagnosis and presentation of a realistic management plan:

"Well I think they've come to see me for advice, for a diagnosis - if they've come for a diagnosis, they've come for treatment, they want to get better and I think it's my responsibility to follow those things through ... so that I see my role as being able to direct them, to stand alongside them really, and work through the maze of going down the right tracks." (GP L)

This left room for patients to contribute their own expertise, in the form of past experiences, practical considerations, the importance of health in their value system, and their own health beliefs. A third group of GPs held the attitude that doctors know best, by virtue of their experience and medical training:

"I guess that's what we're here for. They don't know the functioning of the body and what actually happens so I guess it's up to us to explain it to them." (GP N)

A further problem identified by GPs was the perceived fallibility of medical knowledge; GPs were hesitant to invoke their medical expertise when this rested on somewhat shaky foundations:

"I don't think the medical profession's got a great track record with back injuries really so if they come up with something that's half way sensible then and it works well I'd consider it, sure." (GP H)

Finally, some GPs expressed a reluctance to take a directive role, based on their belief that patients had both the abilities and the desire to act for themselves:

"I think, given honest and accurate information, most people are good at solving their own problems, or at least having some ideas about how to solve the problems." (GP V)

\section{Discussion}

This study indicates the heterogeneous and complex nature of expertise in general practice. It is important to explore the nature of medical expertise as traditionally, the possession of expertise underpins the moral obligation of beneficence ${ }^{5}$; doctors are obliged to act for the good of their patients because doctors alone have access to the specialised knowledge so to act. However, the data from this study questions the importance of the role of scientific or medical knowledge as such, compared with knowledge from other sources, in understanding the nature of medical expertise. 
Certainly the patients in this study recognised medical knowledge and were glad to draw upon such expertise for the treatment of their problems; yet these patients also saw an important role for less scientific knowledge. This included their own experiential bodily knowledge, to which they alone had access, as well as practical knowledge held by both themselves and their GPs.

\section{Personal knowledge of patients}

Similarly, the GPs in this study also indicated that their medical practice did not rely solely upon medical expertise comprised of scientific, medical knowledge; practical experience with patients was described by the GPs as more useful than knowledge found in formal sources. Reviews to date support this view in that there is little in the way of scientific proof or evidence about the effectiveness of various treatments for low back pain. ${ }^{910}$ General practitioners also highlighted the importance of their personal knowledge of patients; the role of this knowledge may be difficult to define clearly but its importance has been noted by other researchers in general practice. ${ }^{11-13}$

This is an interesting point; if GPs are not expert by virtue of their medical (scientific) knowledge, but because of their practical experience together with their personal knowledge of particular patients, this leaves the way open for patients to be equally expert by virtue of their own personal knowledge and experience. Medical expertise is no longer a secret body of knowledge acquired in medical school, but an accumulation of pieces of often disparate information. As such, it is much easier to share parts of this expertise with patients and equally, to recognise the expertise of patients.

It may be helpful to conceive of this in terms of medical and contextual domains: within the medical domain of diagnosis and treatment, the GP is expert whereas within the personal and contextual domain the patient knows best whether or not the management plan is feasible or this particular treatment works for this person. The relative importance of each domain will vary with the kind of medical practice and the nature of the medical problem; for a patient with a ruptured spleen the medical domain is dominant and the good of that patient is very much dependent upon the medical expertise of her doctors. In this study, both patients and GPs recognised the existence of situations of medical urgency in which the medical domain is dominant, but these did not occur commonly. Rather, this study is typical of much general practice in that patients are not usually urgently unwell and it is often not possible to make a specific diagnosis. ${ }^{14-16}$ This results in the patient domain being correspondingly largei. Thus in general practice, acting for the good of the patient entails a necessary role for the patientes expertise as well as that of the GP.

It is important to note that these domains are

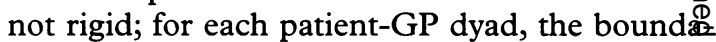
ries were flexible and responsive to the changin situation, so that over time a patient witt recurrent back pain became as expert as his or hèr GP in diagnosing the problem and selecting the appropriate treatment. Similarly, an experience GP could offer helpful insights as to the liket temporal and financial commitments required bet for example, a course of physiotherapy for patient unfamiliar with the system. This flexibilio of domains reflects an important aspect of genera practice, that of medical care occurring over time.

Despite the variable balance between the contextual and the medical domain, medicat expertise played an important and ineliminabe role as a safety net; both patients and GPडs described the importance of ruling out "seriouse causes for patients' symptoms. This was describe $\AA$ by GPs as a filter role; because of their experienge and knowledge, they could see the whole pictures understand things that patients could not, and interpret this as they thought necessary. In the role, the GPs described themselves as knowing better than patients. As we have seen from the results, the GPs in this study varied in the degree to which they ascribed importance to this role The majority were clear about their obligations to act for the patient's good when faced wit situations of potential harm that were square within the medical domain, whilst recognising that much of general practice lies beyond thige medical domain.

\section{Similar attitudes}

One interesting aspect of this study was the degree to which patients and GPs held similar attituden towards medical expertise and knowing best in the consultation. This agreement occurred at number of levels, including the purposive selecs tion by patients of GPs who held similar attitudes to their own. Given this matching, events of the consultation were then influenced by specific feas tures of the problem. Patients for whom management of pain was a priority sought the advice and expertise of the GP; both the problem and the treatment were in the medical domain an management largely drew upon the GP's expers tise. Patients for whom diagnosis was a priority similarly sought the advice and expertise of the GP in order to find out what was wrong, but this did not entail the assumption of managemet 
responsibility by the GP. Management was a further issue to be negotiated as required, with GPs and patients recognising that this involved both medical and non-medical decisions, requiring joint expertise.

This study is subject to certain methodological limitations, in that the patients may not represent a random group, because of their recruitment by participating GPs. This means that the patient group is potentially biased towards those with cordial relationships with their GPs. However, patients did give counter examples deriving from previous medical relationships, adding breadth to the range of experiences described in the interviews. Despite potential methodological limitations, qualitative research of this kind is valuable in that the flexibility of its methods facilitates a depth of understanding absent with quantitative methods. ${ }^{17}$

\section{Conclusion}

From this study we can reach a new understanding of beneficence-in-practice, premised upon an expanded understanding of expertise and a recognition of both medical and contextual domains within general practice consultations. Doctors can and should use their medical expertise for the good of their patients, tempered by the recognition that such expertise is but one element contributing to the overall good of the patient and that in practice such expertise is based as much upon experience as esoteric knowledge. Some patients welcome a doctor who takes charge, and are well able to express this desire whilst others prefer to make their own decisions; the challenge remains for sufficient communication to take place within the consultation to encompass such variety. Empirical investigations such as this have a useful role to play in refining, and it is to be hoped revitalising, ethical concepts.

\section{Acknowledgments}

Financial support for this study was provided by an NH\&MRC scholarship. Thanks to Karen Siegmann for assistance with manuscript preparation.

Wendy A Rogers, PhD, BMBS, DipRacog, MRCGP, $B A H o n s$, is Research Fellow in the Department of General Practice at The Flinders University of South Australia, South Australia.

\section{References}

1 Pellegrino ED, Thomasma DC. For the patient's good: the restoration of beneficence in health care. New York: Oxford University Press, 1988.

2 Beauchamp TL, Childress JF. Principles of biomedical ethics [4th ed]. New York: Oxford University Press, 1994.

3 Frankena WK. Ethics [2nd ed]. New Jersey: Prentice-Hall Inc, 1973.

4 Rothman DJ. Strangers at the bedside. USA: Basic Books, 1991.

5 Sherwin S. No longer patient. Philadelphia: Temple University Press, 1992.

6 Denzin N, Lincoln Y. Handbook of qualitative research. Thousand Oaks CA: Sage, 1994.

7 Miles M, Huberman AM. Qualitative data analysis [2nd ed]. Thousand Oaks CA: Sage, 1994.

8 Dey I. Qualitative data analysis. London: Routledge, 1993.

9 Tulder van M, Koes B, Bouter L. Low back pain in primary care. Amsterdam: EMGO Institute, 1996.

10 Evans G, Richards S. Low back pain: an evaluation of therapeutic interventions. Bristol: Health Care Evaluation Unit, University of Bristol, 1996.

11 Weyrauch $\mathrm{K}$. The personal knowledge of family physicians for their patients. Family Medicine 1994;26:452-5.

12 Rudebeck CE. General Practice and the Dialogue of Clinical Practice. Scandinavian Fournal of Primary Health Care 1993; 2(supp):60-3.

13 Hjortdahl P. Continuity of care: general practitioners' knowledge about and sense of responsibility toward their patients. Family Practice 1992;9,1:3-8.

14 McWhinney I. A textbook of family medicine. New York: Oxford University Press, 1989.

15 Kushner T. Doctor-patient relationships in general practice - a different model. Fournal of Medical Ethics 1981;7:128-31.

16 Pellegrino ED. The healing relationship: the architectonics of clinical medicine. In: Shelp EE, ed. The clinical encounter: the moral fabric of the patient-physician relationship. Dordrecht: D Reidel Publishing Company, 1983.

17 Rogers WA. A systematic review of empirical research into ethics in general practice. British fournal of General Practice 1997; 47:733-7. 